UDC 681.5.011

DOI: $10.18698 / 0236-3933-2019-1-109-128$

\title{
THEORETICAL JUSTIFICATION FOR THE SOLUTION OF THE PROBLEM OF DESTABILIZATION ENERGY-SAVING CONTROL OF MULTIDIMENSIONAL TECHNOLOGICAL OBJECTS OPERATING IN VARIABLE PERFORMANCE CONDITIONS OVER A LONG TIME INTERVAL
}

D.Yu. Muromtsev

A.N. Gribkov

I.V. Tyurin

V.N. Shamkin mdjur@mail.ru

GribkovAlexey@yandex.ru

tyrinilja@yandex.ru

shamkin-v@mail.ru

\section{Tambov State Technical University, Tambov, Russian Federation}

\begin{abstract}
The solution of the so-called "destabilization optimization and destabilization control problems" is analyzed as applied to complex MIMO systems operating in variable performance modes according to the products manufactured over long time intervals. In relation to such problems, an additional economic benefit can be obtained as compared with the traditional method of solving them. Here it is essentially possible to improve the statics of the systems operation over the time interval by determination of such static modes corresponding to the jobs with varying performance so as to achieve the optimal effect in terms of minimizing energy or resources over the considered time interval. In the present paper the previously initiated study was continued, where a certain MIMO system with the load varying repeatedly over a given time interval was considered. There is an integral criterion characterizing the quality of the system operation over the time interval, while the integrand function is a linear parametric one that depends on additional control actions, as well as on the system performance as a parameter. The study is illustrated by the example of a local problem when there is one additional control and one additional state coordinate. The identical problem was derived from the previously formulated linear multi-level one-dimensional problem of destabilization optimization, called the primary problem, after its discussion. Its solution
\end{abstract}

\section{Keywords}

Destabilization optimization, time interval, integral criterion, multidimensional technological object, optimal control 
will allow to analyze the energy-saving control of MIMO systems, ensuring the construction and study of the existence domain of the problem solution and the determination of the function types for the optimal control

Received 04.06.2018

(C) Author(s), 2019

The study was supported by a RFBR grant (project no. 17-08-00457-a)

Introduction. The problems of control as applied to multidimensional technological objects in various subject fields have been discussed in recent years in a number of scientific and technical publications, among which the papers [1-12] should be noted. In particular, the solution of the problem of optimal real time control of a multidimensional linear dynamic object in the class of discrete vector control actions with parallelepiped constraints was considered in Ref. [1]. The methods of optimal control of non-stationary and stationary objects, based on fast dual algorithms for the correction of program solutions, are described. The automatic control system for a certain class of multidimensional objects was designed, and a numerical example for the design of an unstable object control system was given in Ref. [2]. The search for robust control for a certain class of multiply connected dynamic objects was performed in Ref. [3]. The problem of formulating a nonlinear robust control law for a multiply connected dynamic object with the distinctive feature of nonlinearity in control of its mathematical model, is considered. The work [4] is devoted to solving the problem of ensuring the operation of the running multiproduct manufacturing in case of new product introduction, which in terms of the presented article is equivalent to the shift in the product mix and performance of the production.

A discussion of the method and results of modeling a multiply connected autonomous invariant digital system for controlling the process of ammonia synthesis was carried out in Ref. [5], where the consistency and efficiency of the proposed method was proved by modeling. The method of synthesis of automatic regulators for nonstationary linear multidimensional systems was proposed in Ref. [6], the effective allocation of manufacturing process resources using genetic algorithms - in Ref. [7]. This paper discusses the coordination concept that construes as an action of making the right allocation of jobs, resources and control measures to meet the production goal. The use of multidimensional Taylor networks for optimal control of MIMO systems, which are multiple-input and multiple-output nonlinear systems with rough models or even without ones, was demonstrated in Ref. [8].

Various aspects of solving the problem of destabilization energy-saving control of a multidimensional technological object under the conditions of change in jobs by its performance were investigated in Ref. [9-12]. The methodology for 
the complete analysis of energy-saving control of multidimensional objects is presented in Ref. [9], and the methodology for developing systems for destabilization control of technological objects operating under variable load - in Ref. [10]. General issues of destabilization of the operation modes for a multidimensional technological object under the conditions of repeated changes in its performance according to the jobs over a long time interval are contained in Ref. [11]. Here a list of problems to be solved is formulated and a solution is obtained for the case when the object's performance changes twice during the time interval. A technological object with an integral criterion of the operating quality, in which the integrand function linearly depends on additional control actions and also on its load, was considered in Ref. [12]. It is noted that the problem can be decomposed into a set of linear multilevel one-dimensional problems of destabilization optimization.

In this paper, the identical linear multilevel one-dimensional problem formulated in Ref. [12] is analyzed from the perspective of using its results to develop an algorithm of destabilization control of a multidimensional technological object.

Formalized statement of the problem. The considered problem can be conditionally represented as shown in figure [12]. Here, $\mu(0), \mu\left(t_{1}\right), \mu\left(t_{2}\right), \ldots$, $\mu\left(t_{N}\right)=\mu(T)$ are values of the object's state coordinate $\mu(t)$ at the instants of time $t_{0}=0, t_{1}, t_{2}, \ldots, t_{N}=T$ of changing the values $f_{1}, f_{2}, \ldots, f_{N}$ of disturbing action (job) $f ; \omega_{1}, \omega_{2}, \ldots, \omega_{N}$ are components of destabilization control $\omega$ at the time intervals $\left(t_{i}-t_{i-1}\right), i=1, \ldots, n$ (control deviation $u(t)$ from stabilizing control $\stackrel{\circ}{u}(t)$, i.e., $\omega(t)=u(t)-\stackrel{\circ}{u}(t))$.

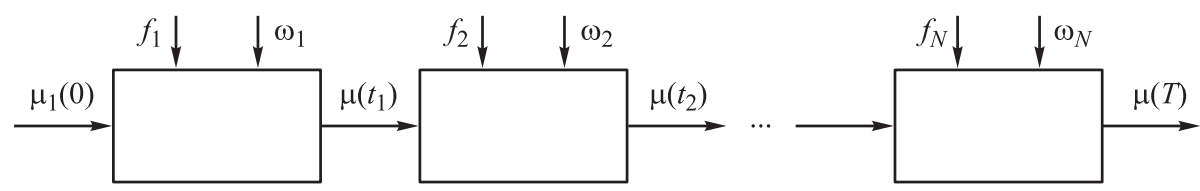

Mnemonic diagram of a multilevel one-dimensional destabilization optimization problem

Let us introduce the following concepts.

A function $x(t)$ is called $n$-level on an interval $[0, \tau]$, if it satisfies the relations

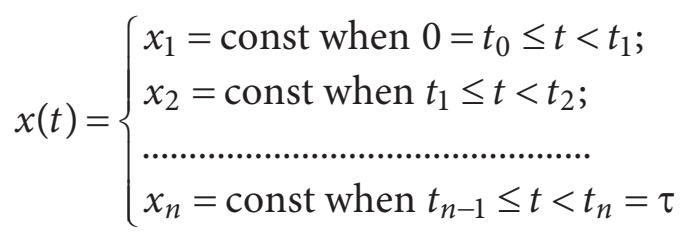


and this function is denoted by $x(t)=\left(x_{1}, x_{2}, \ldots, x_{n}, t_{1}, t_{2}, \ldots, t_{n}\right)$ or $x(t)=(\vec{x}, \vec{t})$, where $\vec{x}=\left(x_{1}, x_{2}, \ldots, x_{n}\right) ; \vec{t}=\left(t_{1}, t_{2}, \ldots t_{n}\right)$.

We assume that the disturbing $f(t)$, control $\omega(t)$ and discrete control $\Delta \mu$ actions are periodic $n$-level functions on the period $T$ :

$$
\begin{aligned}
& f(t)=(\vec{f}, \vec{t}), \omega(t)=(\vec{\omega}, \vec{t}), \Delta \mu(t)=(\Delta \vec{\mu}, \vec{t}), \\
& f(t)=f(T+t) ; \omega(t)=\omega(T+t) ; \Delta \mu(t)=\Delta \mu(T+t),
\end{aligned}
$$

where $T$ characterizes a certain calendar time interval (quarter, year) during which a change in jobs by performance can occur.

Let us use the following notations:

1) $\Delta \mu_{i}, \quad i=1, \ldots, n-$ the discrete control action equal to the change in the state coordinate $\mu(t)$ over the time $\left(t_{i}-t_{i-1}\right)$ as a result of the control action $\omega_{i}$;

2) $\Delta \mu_{i}^{l}, \Delta \mu_{i}^{u p}, \quad i=1, \ldots, n-$ lower and upper permissible values of the discrete control action $\Delta \mu_{i}$;

3) $\mu^{l}, \mu^{u p}-$ lower and upper permissible values of the state coordinate;

4) $\mu_{0}$ - initial state coordinate value;

5) $\mu^{*}(0)$ - optimal value of the state coordinate at the initial instant of time $t=0$;

6) $I(f, \Delta \mu)$ - the functional characterizing the object control efficiency over the time interval $[0, T]$;

7) $A-$ calculated coefficient included in the functional $I$;

8) $a_{i}, b_{i} \quad i=1, \ldots, n-$ the coefficients in the functional $I$, that are at each instant of time $t_{i}$ the functions of the disturbing action value $f_{i}$ at this instant of time.

Identical linear multilevel one-dimensional problem of destabilization optimization. For a given disturbing action $f(t)=(\vec{f}, \vec{t})$ with a period of disturbance $T$ find the control $\Delta \mu^{\star}=\left(\Delta \vec{\mu}^{\star}, \vec{t}\right)$ and the initial value of the state coordinate $\mu^{*}(0)$, at which the functional takes the minimum value

$$
I(f, \Delta \mu)=A+\sum_{i=1}^{n} \frac{b_{i}}{\beta_{i}} \Delta \mu_{i},
$$

the following conditions are met

$$
\begin{gathered}
\Delta \mu_{i}^{l} \leq \Delta \mu_{i} \leq \Delta \mu_{i}^{u p}, i=1, \ldots, n ; \\
\mu^{l} \leq \mu\left(t_{i}\right) \leq \mu^{u p}, i=1, \ldots, n,
\end{gathered}
$$

and the following equation takes place

112 ISSN 0236-3933. Вестник МГТУ им. Н.Э. Баумана. Сер. Приборостроение. 2019. № 1 


$$
\mu(0)=\mu_{0}=\mu(T)
$$

where

$$
\mu\left(t_{i}\right)=\mu_{0}+\sum_{j=1}^{i} \Delta \mu_{j}, i=1, \ldots, n .
$$

Analysis of the problem. From the formula (5) $\mu\left(t_{i+1}\right)=\mu\left(t_{i}\right)+\Delta \mu_{i+1}$, $i=0, \ldots, n-1$, or taking into account the notation $\mu\left(t_{i}\right)=\mu_{i}$,

$$
\mu_{i+1}=\mu_{i}+\Delta \mu_{i+1}, i=0, \ldots, n-1 \text {. }
$$

Let the initial value $\mu_{0}$ is given, at that from the condition (4) the final value $\mu_{n}=\mu_{0}$.

We construct a set of attainability, i.e., for each $i$ we construct a set $\left\{\mu_{i-1}\right\}$, from which one can get to a point $\mu_{n}=\mu_{0}$ by using controls $\Delta \mu_{i}$, $\Delta \mu_{i+1}, \ldots, \Delta \mu_{n}$. The lower $\left(\underline{\mu}_{g}^{i}\right)$ and the upper $\left(\bar{\mu}_{g}^{i}\right)$ bounds are determined by the formulas

$$
\begin{aligned}
& \bar{\mu}_{g}^{i}=\mu_{0}-\sum_{j=i}^{n} \Delta \mu_{i}^{l}, i=n-1, \ldots, 0 ; \\
& \underline{\mu}_{g}^{i}=\mu_{0}-\sum_{j=i}^{n} \Delta \mu_{i}^{u p}, i=n-1, \ldots, 0,
\end{aligned}
$$

or

$$
\begin{aligned}
& \bar{\mu}_{g}^{i}=\mu_{g}^{i+1}-\Delta \mu_{i}^{l} ; \\
& \underline{\mu}_{g}^{i}=\mu_{g}^{i+1}-\Delta \mu_{i}^{u p} .
\end{aligned}
$$

At that $\mu_{i} \in\left[\underline{\mu}_{g}^{i}, \bar{\mu}_{g}^{i}\right]$, or

$$
\underline{\mu}_{g}^{i} \leq \mu_{i} \leq \bar{\mu}_{g}^{i}
$$

Violation of (9) leads to the impossibility of attaining the point $\mu_{n}=\mu_{0}$, i.e., to the violation of condition (4).

We introduce the following terminology. We call the adoption of controls $\Delta \mu_{i}, \ldots, \Delta \mu_{n}(i-n)$ is a strategy, and the fulfillment of the condition $\mu_{n}=\mu_{0}$ is an attaining the point $\mu_{0}$.

Theorem 1. If at the point $t=t_{i}, \mu_{i}=\underline{\mu}_{g}^{i}$ (or $\left.\mu_{i}=\bar{\mu}_{g}^{i}\right)$, then there is a unique $(i-n)$-strategy, at which there will be an attaining the point $\mu_{0}$. This strategy is to move along the bound of the areas of attainability

$$
\mu_{j}=\underline{\mu}_{g}^{j}, j=i, \ldots, n,
$$


or

$$
\mu_{j}=\bar{\mu}_{g}^{j}, j=i, \ldots, n
$$

At that

$$
\Delta \mu_{j}=\underline{\mu}_{g}^{j}-\underline{\mu}_{g}^{j-1}, j=i+1, \ldots, n, \text { if } \mu_{i}=\underline{\mu}_{g}^{i},
$$

or

$$
\Delta \mu_{j}=\bar{\mu}_{g}^{i}-\bar{\mu}_{g}^{j-1}, j=i+1, \ldots, n, \text { if } \mu_{i}=\bar{\mu}_{g}^{i}
$$

4 Let at some point $i$ the value of $\mu_{i}=\underline{\mu}_{i}^{g}$. From (6) it follows that for a point $i+1$ we have

$$
\mu_{i+1}=\underline{\mu}_{i}^{g}+\Delta \mu_{i+1}
$$

It is obvious that the relation $\Delta \mu_{i+1}>\underline{\mu}_{i+1}^{g}-\underline{\mu}_{i}^{g}$ is impossible, since in this case, according to (12), $\Delta \mu_{i+1}>\Delta \mu^{u p}$. The relation $\Delta \mu_{i+1}<\underline{\mu}_{i+1}^{g}-\underline{\mu}_{i}^{g}$ is also impossible, since in this case from (13) we have $\mu_{i+1}<\underline{\mu}_{i}^{g}+\underline{\mu}_{i+1}^{g}-\mu_{i}^{g}$ and $\mu_{i+1}<\mu_{i+1}^{g}$. Because $\mu_{i+1}$ falls outside the area of attainability (9) and it is impossible to attain the point $\mu_{0}$. Hence, for each $j>i$ it is true that $\Delta \mu_{j}=\underline{\mu}_{j}^{g}-\underline{\mu}_{j-1}^{g}, \mu_{j}=\mu_{j}^{g}$.

The assertions (10), (12) of the theorem are proved.

The assertions (11), (13) of the theorem are proved in a similar way.

Corollary 1. If at points $i-1, i$ the following relations are correct:

$$
\begin{array}{lll}
\mu_{i-1} \neq \underline{\mu}_{g}^{i-1}, & \text { or } & \mu_{i-1} \neq \bar{\mu}_{g}^{i-1}, \\
\mu_{i-1}=\underline{\mu}_{g}^{i} & & \mu_{i-1}=\bar{\mu}_{g}^{i},
\end{array}
$$

then there is no point $k<i$, where $\mu_{k}=\mu_{g}^{k}$ or $\mu_{n}=\bar{\mu}_{g}^{k}$.

$\varangle$ The proof follows directly from the assertion of the Theorem 1.

Corollary 2. At point $i=n$ the condition $\mu_{n}=\bar{\mu}_{g}^{n}=\underline{\mu}_{g}^{n}=\mu_{0}$ is correct.

4 The proof clearly follows from (7).

Corollary 3. For $i<k$ inequality $\underline{\mu}_{i}^{g}<\mu_{i}<\mu_{i}^{g}$ holds.

4 The proof follows from Corollary 1.

We denote by $M_{1}^{i}$ the set of admissible $\Delta \mu_{i}$, satisfying to the expression (2), and by $M_{2}^{i}$ the set $\Delta \mu_{i}$, under which the expression (3) is satisfied over the 
Theoretical Justification for the Solution of the Problem of Destabilization Energy-Saving Control...

interval $\left[t_{i-1}, t_{i}\right]$. According to Theorem 2 given in Ref. [12], this is equivalent to the requirement $\mu^{l} \leq \mu_{i-1}+\Delta \mu_{i} \leq \mu^{u p}$, or

$$
\mu^{l}-\mu_{i-1} \leq \mu_{i} \leq \mu^{u p}-\mu_{i-1} .
$$

We denote by $M_{3}^{i}$ the set of values of control actions $\Delta \mu_{i}$, under which conditions (4) are satisfied. According to (9), this set is determined by the relations

$$
\underline{\mu}_{g}^{i} \leq \mu_{i-1}+\Delta \mu_{i} \leq \bar{\mu}_{g}^{i}
$$

or

$$
\mu_{g}^{i}-\mu_{i-1} \leq \Delta \mu_{i} \leq \mu_{g}^{i}-\mu_{i-1} .
$$

The set of controls $M^{i}$, that ensure the fulfillment of the conditions (2)-(4), we determine by the set intersection

$$
M^{i}=M_{1}^{i} \cap M_{2}^{i} \cap M_{3}^{i} .
$$

Next, we consider two practical cases.

The first case. The control actions $\Delta \mu_{i}$ (or $\omega_{i}$ ) are large and the final value $\mu_{\text {fin }}=\mu_{0}$ is always attainable. At that, the range $M^{i}$ of possible values of control actions is characterized by the inequality

$$
\mu^{l}-\mu_{i-1} \leq \Delta \mu_{i} \leq \mu^{u p}-\mu_{i-1}
$$

and is determined as $M^{i}=M_{2}^{i}$.

The second case. The $\mu^{l}$ and $\mu^{u p}$ are large and conditions (3) do not impose limitations on the choice of admissible controls, at that

$$
M^{i}=M_{1}^{i} \cap M_{3}^{i}
$$

and the variation range is determined by the relations

$$
\begin{aligned}
& \Delta \mu_{i} \in\left[\Delta \mu_{i}^{\min }, \Delta \mu_{i}^{\max }\right] ; \\
& \Delta \mu_{i}^{\min }=\max \left[\Delta \mu_{i}^{l}, \underline{\mu}_{g}^{i}-\mu_{i-1}\right] ; \\
& \Delta \mu_{i}^{\max }=\min \left[\Delta \mu_{i}^{u p}, \bar{\mu}_{g}^{i}-\mu_{i-1}\right] .
\end{aligned}
$$

Relations (20) can be conveniently represented as

$$
\begin{aligned}
& \Delta \mu_{i}^{\min }=\Delta \mu_{i}^{l} \underline{\vartheta}_{i}+\left(\underline{\mu}_{g}^{i}-\mu_{i-1}\right)\left(1-\underline{\vartheta}_{i}\right) ; \\
& \Delta \mu_{i}^{\max }=\Delta \mu_{i}^{u p} \bar{\vartheta}_{i}+\left(\bar{\mu}_{g}^{i}-\mu_{i-1}\right)\left(1-\bar{\vartheta}_{i}\right),
\end{aligned}
$$




$$
\begin{aligned}
& \bar{\vartheta}_{i}=\left\{\begin{array}{l}
1, \text { if } \Delta \mu_{i}^{l} \underline{\vartheta}_{i}>\underline{\mu}_{g}^{i}-\mu_{i-1} ; \\
0, \text { if } \Delta \mu_{i}^{u p} \bar{\vartheta}_{i} \leq \bar{\mu}_{g}^{i}-\mu_{i-1},
\end{array}\right. \\
& \underline{\vartheta}_{i}=\left\{\begin{array}{l}
1, \text { if } \Delta \mu_{i}^{l}<\mu_{g}^{i}-\mu_{i-1} ; \\
0, \text { if } \Delta \mu_{i}^{l} \geq \mu_{g}^{i}-\mu_{i-1} .
\end{array}\right.
\end{aligned}
$$

Theorem 2 (the main). Let the disturbing action $f=(\vec{f}, \vec{t})$, where $\vec{f}=\left(f_{1}, f_{2}, \ldots, f_{n}\right), \vec{t}=\left(t_{1}, t_{2}, \ldots, t_{n}\right)$, is a n-level periodic function with a period of $T$ and also let the constraints (3) and (4) are the defining ones among the constraints (2)-(4) of problem (1)-(5), i.e., the variation range $\Delta \mu_{i}$ is defined by the relations

$$
\begin{aligned}
& \mu^{l}-\mu_{i-1} \leq \mu_{i} \leq \mu^{u p}-\mu_{i-1}, i=1, \ldots, n ; \\
& \mu(0)=\mu(T) .
\end{aligned}
$$

Then there is an optimal value $\mu^{*}(0)$ and an optimal n-level control $\omega^{*}=(\vec{\omega}, \vec{t})$, defined as follows:

$$
\begin{aligned}
& \omega_{i}^{*}=\Delta \mu_{i}^{*} / \beta_{i}\left(t_{i}-t_{i-1}\right), \quad i=1, \ldots, n ;
\end{aligned}
$$

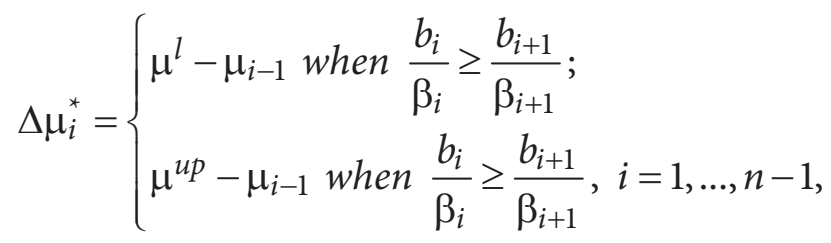

$$
\begin{aligned}
& \Delta \mu_{0}^{*}=\left\{\begin{array}{l}
\mu^{l}, \text { if } \quad \frac{b_{n}}{\beta_{n}} \geq \frac{b_{1}}{\beta_{1}} \\
\mu^{u p}, \text { if } \quad \frac{b_{n}}{\beta_{n}} \geq \frac{b_{1}}{\beta_{1}}
\end{array}\right. \\
& \Delta \mu_{n}^{*}=\mu_{0}-\mu_{n-1}^{*} .
\end{aligned}
$$

According to Theorem 2, given in Ref. [12], the optimal solution of problem (1)-(5) in the form of a $n$-level function $\omega(t)=(\vec{\omega}, \vec{t})$ exists.

We introduce the Bellman function $B_{i}\left(\mu_{i-1}\right)$, representing the minimum value of the sum $\sum_{j=1}^{n}\left(b_{j} / \beta_{j}\right) \Delta \mu_{j}$, obtained when choosing admissible $(i-n)$-strategies 


$$
B_{i}\left(\mu_{i-1}\right)=\min _{\substack{\mu_{i}^{l}-\mu_{i-1} \leq \Delta \mu_{i} \leq \mu_{i}^{u p}-\mu_{i-1} \\ \mu_{i=1}^{l}-\mu_{i} \leq \Delta \mu_{i+1} \leq \mu_{i+1}^{u p}-\mu_{i-1}}} \sum_{j=1}^{n}\left(b_{j} / \beta_{j}\right) \Delta \mu_{j}
$$

at that, due to the necessity to fulfill the periodicity condition (4), we have

$$
\mu_{n}=\mu_{0} .
$$

The functional equations that relates the Bellman functions (28) by recurrence relationships, have the following form:

$$
\begin{gathered}
B_{n}\left(\mu_{n-1}\right)=\frac{b_{n}}{\beta_{n}}\left(\mu_{0}-\mu_{n-1}\right), \\
B_{i}\left(\mu_{i-1}\right)=\min _{\mu_{i}^{l}-\mu_{i-1} \leq \Delta \mu_{i} \leq \mu_{i}^{u p}-\mu_{i-1}}\left[\frac{b_{i}}{\beta_{i}} \Delta \mu_{i}+B_{i+1}\left(\mu_{i}\right)\right], i=n-1, \ldots, 1 .
\end{gathered}
$$

By the method of mathematical induction, we prove that Bellman functions (28) are representable for any $i=1, \ldots, n-1$ in the form of

$$
B_{i}\left(\mu_{i-1}\right)=A_{i}-\mu_{i-1} \frac{b_{i}}{\beta_{i}}+\frac{b_{n}}{\beta_{n}} \mu_{0}
$$

where

$$
A_{i}=A_{i+1}+\tilde{\mu}_{i}\left(\frac{b_{i}}{\beta_{i}}-\frac{b_{i-1}}{\beta_{i-1}}\right)
$$

and

$$
\tilde{\mu}_{i}=\left\{\begin{array}{l}
\mu^{u p}, \text { if } \frac{b_{i}}{\beta_{i}}<\frac{b_{i-1}}{\beta_{i-1}} \\
\mu^{l}, \text { if } \frac{b_{i}}{\beta_{i}} \geq \frac{b_{i-1}}{\beta_{i-1}} .
\end{array}\right.
$$

Let for some $i=s+1$ the Bellman function has the form of (31), (32), i.e.,

$$
\begin{aligned}
& B_{s+1}\left(\mu_{s}\right)=A_{s+1}-\mu_{s} \frac{b_{s+1}}{\beta_{s+1}}+\frac{b_{n}}{\beta_{n}} \mu_{0}, \\
& A_{s+1}=A_{s+2}+\mu_{s+1}\left(\frac{b_{s+1}}{\beta_{s+1}}-\frac{b_{s}}{\beta_{s}}\right) .
\end{aligned}
$$

Let us prove that also for $i=s$ the Bellman function $B_{s}\left(\mu_{s-1}\right)$ also has the form of (31), (32). 
According to (30), we have

$$
B_{s}\left(\mu_{s-1}\right)=\min _{\mu_{s}^{l}-\mu_{s-1} \leq \Delta \mu_{s} \leq \mu_{s}^{u p}-\mu_{s-1}}\left[\frac{b_{s}}{\beta_{s}} \Delta \mu_{s}+B_{s+1}\left(\mu_{s-1}-\Delta \mu_{s}\right)\right] .
$$

Substituting (31) into (34) and converting the resulting expression, we have

$$
B_{s}\left(\mu_{s-1}\right)=\min _{\mu_{s}^{l}-\mu_{s-1} \leq \Delta \mu_{s} \leq \mu_{s}^{u p}-\mu_{s-1}}\left[\Delta \mu_{s}\left(\frac{b_{s}}{\beta_{s}}-\frac{b_{s+1}}{\beta_{s+1}}\right)+\mu_{0} \frac{b_{n}}{\beta_{n}}-\mu_{s-1} \frac{b_{s+1}}{\beta_{s+1}}+A_{s+1}\right] .
$$

Due to the fact that the expression in square brackets in (35) is linear with respect to $\Delta \mu_{s}$, it is obvious that the optimal value of $\Delta \mu_{s}$ is equal to:

$$
\Delta \mu_{s}^{*}=\left\{\begin{array}{l}
\mu_{s}^{l}-\mu_{s-1}, \text { if }\left(\frac{b_{s}}{\beta_{s}}-\frac{b_{s+1}}{\beta_{s+1}}\right)<0 ; \\
\mu_{s}^{u p}-\mu_{s-1}, \text { if }\left(\frac{b_{s}}{\beta_{s}}-\frac{b_{s+1}}{\beta_{s+1}}\right) \geq 0 .
\end{array}\right.
$$

By introducing the notation

$$
\tilde{\mu}_{s}=\left\{\begin{array}{l}
\mu^{u p}, \text { if }\left(\frac{b_{s}}{\beta_{s}}-\frac{b_{s+1}}{\beta_{s+1}}\right)<0 \\
\mu^{l}, \text { if }\left(\frac{b_{s}}{\beta_{s}}-\frac{b_{S+1}}{\beta_{s+1}}\right) \geq 0
\end{array}\right.
$$

we write (36) in a more general form $\Delta \mu_{s}^{*}=\tilde{\mu}_{s}-\mu_{s-1}$, at that

$$
B_{s}\left(\mu_{s-1}\right)=\left(\tilde{\mu}_{s}-\mu_{s-1}\right)\left(\frac{b_{s}}{\beta_{s}}-\frac{b_{s+1}}{\beta_{s+1}}\right)-\mu_{s-1}+\mu_{0} \frac{b_{s+1}}{\beta_{s+1}}+\mu_{0} \frac{b_{n}}{\beta_{n}}+A_{s+1},
$$

or

$$
B_{s}\left(\mu_{s-1}\right)=A_{s}-\mu_{s-1} \frac{b_{s}}{\beta_{s}}+\mu_{0} \frac{b_{n}}{\beta_{n}}
$$

where

$$
A_{s}=A_{s+1}+\tilde{\mu}_{s}\left(\frac{b_{s}}{\beta_{s}}-\frac{b_{s+1}}{\beta_{s+1}}\right) .
$$

Expression (37) has the form of (31). Thus, by virtue of randomness of $s$, we have the following: if for any $i$ the Bellman function $B_{i}\left(\mu_{i-1}\right)$ is representable in the form of (31), then it is representable in this form also for $B_{i-1}\left(\mu_{i-2}\right)$. 
We show that for $i=n-\mathbf{1}$ the Bellman function $B_{n-1}\left(\mu_{n-2}\right)$ can be represented in the form of (31).

Indeed, considering the expression (30), we have

$$
\begin{aligned}
B_{n-1}\left(\mu_{n-2}\right) & =\min _{\mu^{l}-\mu_{n-2} \leq \Delta \mu_{n-1} \leq \mu^{u p}-\mu_{n-2}}\left[\Delta \mu_{n-1}\left(\frac{b_{n-1}}{\beta_{n-1}}+B_{n}\left(\mu_{n-1}\right)\right)\right]= \\
& =\min _{\mu^{l}-\mu_{n-2} \leq \Delta \mu_{n-1} \leq \mu^{u p}-\mu_{n-2}}\left[\frac{b_{n-1}}{\beta_{n-1}} \Delta \mu_{n-1}-\mu_{0} \frac{b_{n}}{\beta_{n}}-\frac{b_{n}}{\mu_{n}}\left(\mu_{n-2}+\Delta \mu_{n-1}\right)\right]= \\
& =\min _{\mu^{l}-\mu_{n-2} \leq \Delta \mu_{n-1} \leq \mu^{u p}-\mu_{n-2}}\left[\Delta \mu_{n-1}\left(\frac{b_{n-1}}{\beta_{n-1}}-\frac{b_{n}}{\beta_{n}}\right)+\mu_{0} \frac{b_{n}}{\mu_{n}}-\frac{b_{n}}{\mu_{n}} \mu_{n-2}\right] .
\end{aligned}
$$

Using the notation (33), from here we get

$$
B_{n-1}\left(\mu_{n-2}\right)=A_{n-1}-\mu_{n-2} \frac{b_{n-1}}{\beta_{n-1}}+\mu_{0} \frac{b_{n}}{\mu_{n}},
$$

where

$$
A_{n-1}=\tilde{\mu}\left(\frac{b_{n-1}}{\beta_{n-1}}-\frac{b_{n}}{\mu_{n}}\right) .
$$

Since $A_{n}=\mathbf{0}$, then (38), (39) correspond to the form of (31)-(33), i.e., the form of the Bellman function is truly determined by the (31).

However, in this case, the expression in square brackets (35) should be minimized at each step $s$. This expression is linear with respect to $\Delta \mu_{s}$, i.e., its minimum is reached either with $\Delta \mu_{s}=\mu_{s}^{l}-\mu_{s-1}$, or $\Delta \mu_{s}=\mu_{s}^{u p}-\mu_{s-1}$. The selection is carried out in accordance with (36). The assertion (25) of Theorem 2 is proved.

In accordance with (31) the Bellman function for $i=\mathbf{1}$ has the form

$$
B_{1}\left(\mu_{0}\right)=A_{1}-\mu_{0}\left(\frac{b_{1}}{\beta_{1}}-\frac{b_{n}}{\mu_{n}}\right) .
$$

The minimum of this function is equal to the minimum of the functional (1) and is achieved at:

- the maximum value of $\mu_{0}$, if the bracketed expression is positive,

$$
\mu_{0}=\mu^{u p} \text { for } \frac{b_{1}}{\beta_{1}}>\frac{b_{n}}{\beta_{n}} \text {; }
$$

- the minimum value of $\mu_{0}$, if the bracketed expression is negative, 


$$
\mu_{0}=\mu^{l} \text { for } \frac{b_{1}}{\beta_{1}}>\frac{b_{n}}{\beta_{n}} \text {. }
$$

The Theorem 2 is proved.

Theorem 3 (the main). Let the disturbing action $f=(\vec{f}, \vec{t})$, where $\vec{f}=\left(f_{1}, f_{2}, \ldots, f_{n}\right), \vec{t}=\left(t_{1}, t_{2}, \ldots, t_{n}\right)$, is a $n$-level periodic function with a period of $T$ and also let the constraints (2) and (4) are the defining ones among the constraints (2)-(4) of problem (1)-(5), i.e., the variation range $\Delta \mu_{i}$ is defined by the relations

$$
\begin{aligned}
& \Delta \mu_{i}^{l} \leq \Delta \mu_{i} \leq \Delta \mu_{i}^{l}, i=1, \ldots, n, \\
& \mu(0)=\mu(T) .
\end{aligned}
$$

Then there is an optimal value $\mu^{*}(0)$ and an optimal n-level control $\omega^{*}=(\vec{\omega}, \vec{t})$, defined as follows:

$$
\begin{aligned}
& \omega_{i}^{*}=\Delta \mu_{i}^{*} / \beta_{i}\left(t_{i}-t_{i-1}\right), i=1, \ldots, n, \quad \bar{\mu}_{i}^{g}=\mu_{0}-\sum_{j=i+1}^{n} \Delta \mu_{j}^{l}, \quad i=0, \ldots, n-1, \\
& \underline{\mu}_{i}^{g}=\mu_{0}-\sum_{j=i+1}^{n} \Delta \mu_{j}^{u p}, j=0, \ldots, n-1, \\
& \Delta \mu_{i}^{l}=\min \left[\beta_{i} \omega_{i}^{u p}\left(t_{i}-t_{i-1}\right), \beta_{i} \omega_{i}^{l}\left(t_{i}-t_{i-1}\right)\right], \\
& \Delta \mu_{i}^{u p}=\max \left[\beta_{i} \omega_{i}^{u p}\left(t_{i}-t_{i-1}\right), \beta_{i} \omega_{i}^{l}\left(t_{i}-t_{i-1}\right)\right] \text {, } \\
& \Delta \mu_{i}^{*}=\left\{\begin{array}{l}
\bar{\mu}_{i}^{g}-\bar{\mu}_{i-1}^{g}, \text { if } i>\bar{k}+1 ; \\
\bar{\mu}_{i}^{g}-\mu_{i-1}, \text { if } i>\bar{k} ; \\
\underline{\mu}_{i}^{g}-\underline{\mu}_{i-1}^{g}, \text { if } i>\underline{k}+1 ; \\
\underline{\mu}_{i}^{g}-\mu_{i-1}^{g}, \text { if } i=\underline{k} ; \\
\Delta \mu_{i}^{u p}, \text { if } \frac{b_{i}}{\beta_{i}}<\frac{b_{\bar{k}}^{-}}{\beta_{\bar{k}}^{-}}, i<\bar{k} ; \\
\Delta \mu_{i}^{l}, \text { if } \frac{b_{i}}{\beta_{i}} \geq \frac{b_{\bar{k}}}{\beta_{\bar{k}}}, i<\bar{k} ; \\
\Delta \mu_{i}^{l}, \text { if } \frac{b_{i}}{\beta_{i}}<\frac{b_{\underline{k}}}{\beta_{\underline{k}}}, i<\underline{k} ; \\
\Delta \mu_{i}^{l}, \text { if } \frac{b_{i}}{\beta_{i}} \geq \frac{b_{\underline{k}}}{\beta_{\bar{k}}}, i<\underline{k},
\end{array}\right.
\end{aligned}
$$


where

$$
\begin{gathered}
\bar{k}=\arg \min _{j}\left\{\Delta \mu_{j}^{u p} \geq \bar{\mu}_{j}^{g}-\mu_{j-1}\right\} ; \\
\underline{k}=\underset{j}{\arg \min }\left\{\Delta \mu_{j}^{l} \geq \underline{\mu}_{j}^{g}-\mu_{j-1}\right\}, \\
\mu_{0}^{*}=\left\{\begin{array}{l}
\mu^{u p}, \text { if } \frac{b_{\tilde{k}}}{\beta_{\tilde{k}}} \geq \frac{b_{n}}{\beta_{n}} ; \\
\mu^{l}, \text { if } \frac{b_{\tilde{k}}}{\beta_{\tilde{k}}}<\frac{b_{n}}{\beta_{n}}, \\
\tilde{k}=\min (\bar{k}, \underline{k}) .
\end{array}\right.
\end{gathered}
$$

At that $\bar{k}$ or (and) $\underline{k}$ are equal to $n$.

4 We introduce the Bellman function, analogous to (28). We denote the final state $\mu(T)$ as equal to $\mu(0)=\mu_{0}$. At that, the Bellman functional equations, similar to (30), have the form

$$
\begin{aligned}
& B_{n}\left(\mu_{n-1}\right)=\frac{b_{n}}{\beta_{n}}\left(\mu_{0}-\mu_{n-1}\right), \\
& B_{i}\left(\mu_{i-1}\right)=\min _{\Delta \mu_{i} \in M^{i}}\left[\frac{b_{i}}{\beta_{i}} \Delta \mu_{i}+B_{i+1}\left(\mu_{i}\right)\right], i=n-1, \ldots, 1,
\end{aligned}
$$

where $M^{i}$ - the range of acceptable variations $\Delta \mu_{i}$, defining by the relations (21), (22).

Let at the point $\bar{k}$ there are the following relations:

$$
\mu_{k}^{-}=\bar{\mu}_{k}^{g}, \quad \mu_{k-1} \neq \bar{\mu}_{k-1}^{g}
$$

According to Theorem 1 , in this case there is only one $(\bar{k}-n)$-strategy that leads to the point $\mu_{0}$ :

$$
\Delta \mu_{i}=\bar{\mu}_{i}^{g}-\bar{\mu}_{i-1}^{g}, i=k+1, \ldots, n .
$$

For $i=k$ we have $\Delta \mu_{k}^{-}=\bar{\mu} \frac{g}{k}-\bar{\mu} \frac{g}{k-1}$.

The assertions (43a), (43b) of the Theorem 3 are proved.

Let at the point $\bar{k}$ there are the following relations:

$$
\mu_{\bar{k}}^{-}=\mu_{k}^{g}, \quad \mu_{\bar{k}-1} \neq \underline{\mu}_{k-1}^{g} .
$$


According to Theorem 1 , in this case there is only one $(\bar{k}-n)$-strategy that leads to the point $\mu_{0}$ :

$$
\Delta \mu_{i}=\underline{\mu}_{i}^{g}-\mu_{i-1}^{g}
$$

For $i=\underline{k}$ we have $\Delta \mu_{\underline{k}}=\bar{\mu}_{\underline{k}}^{g}-\mu_{\underline{k}-1}^{g}$.

The assertions (43c), (43d) of the Theorem 3 are proved.

We introduce the general notation for $\bar{k}$ and $\underline{k}$. In this case (49)-(52) can be rewritten.

Let at the point $\tilde{k}$ there are the relations $\mu_{\tilde{k}}=\tilde{\mu}_{k}^{g}, \quad \mu_{\tilde{k}-1} \neq \tilde{\mu}_{k-1}^{g}$, where $\tilde{\mu}$ is the general notation for $\bar{\mu}$ and $\underline{\mu}$. According to Theorem 1, at that there is only one $(\tilde{k}-n)$-strategy that leads to the point $\mu_{0}: \Delta \mu_{i}=\tilde{\mu}_{i}^{g}-\mu_{i-1}^{g}$. For $i=\tilde{k}$ we have $\Delta \mu_{\underline{\tilde{k}}}=\tilde{\mu}_{\tilde{k}}^{g}-\mu_{\tilde{\hat{k}}-1}^{g}$.

According to the Corollary 1 of Theorem 1 , the point $\tilde{k}$ is unique. If $\bar{k} \neq n$, then $\underline{k}=n$, if $\underline{k} \neq n$, then $\bar{k}=n$. Obviously, there may be a limiting case when $\underline{k}=\bar{k}=n$.

The assertion (47) of the Theorem 3 is proved.

The Bellman function with $(\tilde{k}-n)$-strategy, if $\tilde{k} \neq n$, for $i=\tilde{k}+1, \ldots, n$ takes the form

$$
\begin{aligned}
& B_{n}\left(\tilde{\mu}_{n-1}^{g}\right)=\frac{b_{n}}{\beta_{n}}\left(\mu_{0}-\tilde{\mu}_{n-1}^{g}\right), \\
& \ldots \ldots \ldots \ldots \ldots \ldots \ldots \ldots \ldots \ldots \ldots \ldots \ldots \ldots \ldots \ldots \ldots \ldots \ldots . . .
\end{aligned}
$$

For $i=\tilde{k}$ we have

$$
B_{\tilde{k}}\left(\tilde{\mu}_{\tilde{k}-1}\right)=A_{\tilde{k}}-\mu_{\tilde{k}-1} \frac{b_{\tilde{k}}}{\beta_{\tilde{k}}}+\frac{b_{n}}{\beta_{n}} \mu_{0}
$$

where

$$
A_{\tilde{k}}=-\frac{b_{n}}{\beta_{n}} \tilde{\mu}_{n-1}^{g}+\sum_{j=\tilde{k}+1}^{n-1} \frac{b_{j}}{\beta_{j}}\left(\tilde{\mu}_{j}^{g}-\tilde{\mu}_{j-1}^{g}\right)+\frac{b_{\tilde{k}}}{\beta_{\tilde{k}}} \tilde{\mu}_{k}^{g} .
$$

For $i<\tilde{k}$ in accordance with Corollary 3 of Theorem 1 the inequalities $\underline{\mu}_{i}^{g}<\mu_{i-1}+\Delta \mu_{i}<\bar{\mu}_{i}^{g}$, or $\underline{\mu}_{i}^{g}-\mu_{i-1}<\Delta \mu_{i}<\bar{\mu}_{i}^{g}-\mu_{i-1}$ are strictly satisfied. At that, the expression (2) becomes the defining constraint, i.e., a set $M^{i}$ in the 
form (19) of the varied values $\Delta \mu_{i}$ becomes equal to the set $M_{1}^{i}$, i.e., $\Delta \mu_{i}^{l} \leq \Delta \mu_{i}<\Delta \mu_{i-1}^{u p}, i=1, \ldots, \tilde{k}-1$.

Let us prove that the Bellman function for $i=1, \ldots, \tilde{k}-1$, has the form

$$
B_{i}\left(\mu_{i-1}\right)=A_{i}-\mu_{i-1} \frac{b_{\tilde{k}}}{\beta_{\tilde{k}}}+\frac{b_{n}}{\beta_{n}} \mu_{0}
$$

where

$$
\begin{aligned}
& A_{i}=A_{i+1}+\left(\frac{b_{i}}{\beta_{i}}-\frac{b_{\tilde{k}}}{\beta_{\tilde{k}}}\right) \Delta \mu_{i}^{*}, \\
& \Delta \mu_{i}^{*}=\left\{\begin{array}{l}
\Delta \mu_{i}^{u p}, \text { if } \frac{b_{i}}{\beta_{i}}<\frac{b_{\tilde{k}}}{\beta_{\tilde{k}}} ; \\
\mu_{i}^{l}, \text { if } \frac{b_{i}}{\beta_{i}} \geq \frac{b_{\tilde{k}}}{\beta_{\tilde{k}}} .
\end{array}\right.
\end{aligned}
$$

Let for some $i=s+\mathbf{1}<\tilde{k}$ the Bellman function be written as

$$
B_{s+1}\left(\mu_{s}\right)=A_{s+1}-\mu_{s} \frac{b_{\tilde{k}}}{\beta_{\tilde{k}}}+\frac{b_{n}}{\beta_{n}} \mu_{0}
$$

Here,

$$
A_{s+1}=A_{s+2}+\left(\frac{b_{s+1}}{\beta_{s+1}}-\frac{b_{\tilde{k}}}{\beta_{n}}\right)+\Delta \mu_{s+1} .
$$

In this case, for $i=s$ in accordance with (48) we get

$$
\begin{gathered}
B_{s}\left(\mu_{s-2}\right)=\min _{\mu_{s}^{l} \leq \Delta \mu_{s} \leq \mu_{s}^{u p}}\left[\frac{b_{s}}{\beta_{s}} \Delta \mu_{s}+A_{s+1}-\left(\mu_{s-1}+\Delta \mu_{s}\right) \frac{b_{\tilde{k}}}{\beta_{\tilde{k}}}+\frac{b_{n}}{\beta_{n}} \mu_{0}\right]= \\
=\min _{\mu_{s}^{l} \leq \Delta \mu_{s} \leq \mu_{s}^{u p}}\left[\Delta \mu_{s}\left(\frac{b_{s}}{\beta_{s}}-\frac{b_{\tilde{k}}}{\beta_{\tilde{k}}}\right)+A_{s+1}-\mu_{s-1} \frac{b_{\tilde{k}}}{\beta_{\tilde{k}}}+\frac{b_{n}}{\beta_{n}} \mu_{0}\right] .
\end{gathered}
$$

Since the expression in square brackets in (57) linearly depends on $\Delta \mu_{s}$, the optimal value of $\Delta \mu_{s}^{*}$ is determined by the expression

$$
\Delta \mu_{s}^{*}=\left\{\begin{array}{l}
\Delta \mu_{s}^{u p}, \text { if } \frac{b_{s}}{\beta_{s}}<\frac{b_{\tilde{k}}}{\beta_{\tilde{k}}} ; \\
\Delta \mu_{s}^{l}, \text { if } \frac{b_{s}}{\beta_{s}} \geq \frac{b_{\tilde{k}}}{\beta_{\tilde{k}}} .
\end{array}\right.
$$


At that

where

$$
B_{s}\left(\mu_{s-1}\right)=A_{s}-\mu_{s-1} \frac{b_{\tilde{k}}}{\beta_{k}}+\frac{b_{n}}{\beta_{n}} \mu_{0}
$$

i.e., coincides with (56).

$$
A_{s}=A_{s+1}+\Delta \mu_{s}^{*}\left(\frac{b_{s}}{\beta_{s-1}}-\frac{b_{\tilde{k}}}{\beta_{\tilde{k}}}\right),
$$

If for some $i<\tilde{k}$ the Bellman function has the form (56), then it has the same form as for $i-1$.

Let us find the Bellman function for $i=\tilde{k}-1$.

According to (48), we have

$$
\begin{gathered}
\mathrm{B}_{\tilde{k}-1}\left(\mu_{\tilde{k}-2}\right)=\min _{\mu_{\tilde{k}-1}^{l} \leq \Delta \mu_{\tilde{k}-1} \leq \mu_{\tilde{k}-1}^{u p}}\left[\frac{b_{\tilde{k}-1}}{\beta_{\tilde{k}-1}}+\mathrm{B}_{\tilde{k}}\left(\mu_{\tilde{k}-1}\right)\right]= \\
=\min _{\mu_{\tilde{k}-1}^{l} \leq \Delta \mu_{\tilde{k}-1} \leq \mu_{\tilde{k}-1}^{u p}}\left[\frac{b_{\tilde{k}-1}}{\beta_{\tilde{k}-1}} \Delta \mu_{\tilde{k}-1}+A_{\tilde{k}}-\mu_{\tilde{k}-1} \frac{b_{\tilde{k}}}{\beta_{\tilde{k}}} \mathrm{~B} \frac{b_{n}}{\mu_{n}} \mu_{0}\right]= \\
=\min _{\mu_{\tilde{k}-1}^{l} \leq \Delta \mu_{\tilde{k}-1} \leq \mu_{\tilde{k}-1}^{u p}}\left[\Delta \mu_{\tilde{k}-1}\left(\frac{b_{\tilde{k}-1}}{\beta_{\tilde{k}-1}}-\frac{b_{\tilde{k}}}{\beta_{\tilde{n}}}\right)+A_{\tilde{k}}-\mu_{\tilde{k}-2} \frac{b_{\tilde{k}}}{\beta_{\tilde{k}}}+\frac{b_{n}}{\beta_{n}} \mu_{0}\right] .
\end{gathered}
$$

Hence,

$$
B_{\tilde{k}-1}\left(\mu_{\tilde{k}-2}\right)=A_{k-1}-\mu_{\tilde{k}-2} \frac{b_{\tilde{k}}}{\beta_{k}}+\frac{b_{n}}{\beta_{n}} \mu_{0} .
$$

Because $B_{\tilde{k}-1}\left(\mu_{\tilde{k}-2}\right)$ satisfies (56), as well as due to the previously proved proposition (if $B_{i}\left(\mu_{i-1}\right)$ has the form of (56), then $B_{i-1}\left(\mu_{i-2}\right)$ also has the form of (56)), we obtain: for all $i=1, \ldots, k-1$ the function $B_{i}\left(\mu_{i-1}\right)$ has the form of (56).

However, in accordance with (58), this means that the optimal value of the control $\Delta \mu_{i}^{*}$ at each step $i=1, \ldots, k-1$ is determined by the expression

$$
\Delta \mu_{i}^{*}=\left\{\begin{array}{l}
\Delta \mu_{i}^{u p}, \text { if } \frac{b_{i}}{\beta_{i}}<\frac{b_{\tilde{k}}}{\beta_{\tilde{k}}} \\
\Delta \mu_{i}^{l}, \text { if } \frac{b_{i}}{\beta_{i}} \geq \frac{b_{\tilde{k}}}{\beta_{\tilde{n}}} .
\end{array}\right.
$$

The assertions (43e)-(43h) of the Theorem 3 are proved.

The Bellman function for $i=\mathbf{1}$ according to (56)

$$
B_{1}\left(\mu_{0}\right)=A_{1}-\mu_{0} \frac{b_{\tilde{k}}}{\beta_{\tilde{k}}}+\frac{b_{n}}{\beta_{n}} \mu_{0}=A_{1}-\mu_{0}\left(\frac{b_{\tilde{k}}}{\beta_{\tilde{k}}}-\frac{b_{n}}{\beta_{n}}\right)
$$


and reaches a minimum at

$$
\Delta \mu_{0}=\left\{\begin{array}{l}
\mu^{u p}, \text { if } \frac{b_{\tilde{k}}}{\beta_{\tilde{k}}} \geq \frac{b_{n}}{\beta_{n}} ; \\
\Delta \mu^{l}, \text { if } \frac{b_{\tilde{k}}}{\beta_{\tilde{k}}}<\frac{b_{n}}{\beta_{n}} .
\end{array}\right.
$$

The Theorem 3 is proved.

Corollary 1. The value of $\bar{k}$ satisfies the condition $\bar{k} \geq m$, where $m$ is determined from the equation

$$
\bar{m}=\underset{j}{\arg \min }\left(\sum_{l=1}^{j} \Delta \mu_{l}^{u p} \geq \bar{\mu}_{j}^{g}\right) .
$$

$\varangle$ It is obvious, that always $\mu_{j} \leq \sum_{l=1}^{j} \Delta \mu_{l}^{u p}$ and in that case, if $\sum_{l=1}^{j} \Delta \mu_{l}^{u p}<\bar{\mu}_{j}^{g}$, the relation $\mu_{j}<\bar{\mu}_{l}^{g}$ always takes place.

Corollary 2. The value of $\underline{k}$ satisfies the condition $\underline{k} \geq \underline{m}$, where $\underline{m}$ is determined from the equation

$$
\underline{m}=\underset{j}{\arg \min }\left(\sum_{l=1}^{j} \Delta \mu_{l}^{l} \leq \mu_{j}^{g}\right) .
$$

There is always a relation $\mu_{j} \geq \sum_{l=1}^{j} \Delta \mu_{l}^{l}$. It follows from this that if $\underline{\mu}_{j}^{g}<\sum_{l=1}^{j} \Delta \mu_{l}^{l}$, then always $\mu_{j}>\underline{\mu}_{l}^{g}$.

Conclusion. A theoretical justification of the solution of the identical linear $n$-level one-dimensional problem of destabilization optimization (1)-(5) was performed in the form of a proof of Theorems 1-3 and their corollaries, which allowed us to obtain analytical expressions for the corresponding components of control actions and state coordinate of the object.

The solution of this problem (the choice of the optimal combination of control actions and the state coordinate corresponding to the $n$-level disturbing action on the time interval), determined by the main theorems 2 and 3, was obtained for two important practical cases.

The first case. It is the case when the control actions $\Delta \mu_{i}$ (or $\left.\omega_{i}\right)$ are large at each solution stage and the final value $\mu_{f i n}=\mu_{0}$ is always attainable for the state coordinate. This case corresponds to a situation in which there is a small storage container within the technological object, where a liquid can accumu- 
D.Yu. Muromtsev, A.N. Gribkov, I.V. Tyurin, V.N. Shamkin

late or deteriorate as a result of destabilization of the mode. At that only two constraints remain in the formulation of the problem of destabilization optimization:

$$
\begin{aligned}
& \mu^{l} \leq \mu\left(t_{i}\right) \leq \mu^{u p}, i=0, \ldots, n ; \\
& \mu(0)=\mu_{0}=\mu(T) .
\end{aligned}
$$

The second case. It is the case when $\mu^{l}$ and $\mu^{u p}$ are large and condition (3) does not impose constraints on the choice of admissible controls, and the initial value of $\mu(0)$ does not matter. This case corresponds to the situation with large storage container. At that, a set of constraints for the problem of destabilization optimization has the form

$$
\begin{aligned}
& \Delta \mu_{i}^{u p} \leq \Delta \mu_{i} \leq \Delta \mu_{i}^{l}, i=1, \ldots, n ; \\
& \mu(0)=\mu_{0}=\mu(T) .
\end{aligned}
$$

Expressions (24)-(27) and (42), (60), (61) for the optimal value of the state coordinate $\mu^{*}(0)$ and optimal $n$-level control $\omega^{*}=(\vec{\omega}, \vec{t})$, contained in Theorems 2 and 3, as well as relations (60) and (61) in the corollaries of the Theorem 3 , are the solution of the identical linear $n$-level one-dimensional problem of destabilization optimization (1)-(5). They constitute the basis of the algorithm of the destabilization control for multidimensional technological objects that operate when their performance repeatedly changes over a long time interval, when they have several opportunities to introduce a destabilization mode.

An interesting and important practical case is when an additional coordinate of the state of a technological object, caused by the introduction of a destabilizing factor, is affected not only by an additional control action, but also by other controls (as if there is an "excess of controls"). This case deserves a separate study.

Translated by A. Kurnosenko

\section{REFERENCES}

[1] Gabasov R., Kirillova F.M., Ha V.T.T. Optimal real-time control of multidimensional dynamic plant. Autom. Remote Control, 2015, vol. 76, iss. 1, pp. 98-110.

DOI: $10.1134 / S 0005117915010099$

[2] Gayduk A.R., Kolokolova K.V. Synthesis of control systems by unstable multivariable plants. Nauchnyy Vestn. Novosib. Gos. Tekh. Univ. [Science Bulletin of The Novosibirsk State Technical University], 2017, no. 1 (66), pp. 26-40 (in Russ.).

DOI: 10.17212/1814-1196-2017-1-26-40

126 ISSN 0236-3933. Вестник МГТУ им. Н.Э. Баумана. Сер. Приборостроение. 2019. № 1 
Theoretical Justification for the Solution of the Problem of Destabilization Energy-Saving Control...

[3] Eremin E.L., Shelenok E.A. Robust control for one class of multivariable dynamic plants. Autom. Remote Control, 2017, vol. 78, iss. 6, pp. 1046-1058.

DOI: $10.1134 / S 0005117917060066$

[4] Karpushkin S.V., Krasnyanskiy M.N., Borisenko A.B. Optimization of existing equipment for multiproduct batch plants in new product release. Vestnik TGTU [Transactions of the TSTU], 2016, vol. 22, no. 2, pp. 238-254 (in Russ.).

[5] Kudryashov V.S., Tikhomirov S.G., Ryazantsev S.V., et al. A method of simulation of a multilift digital system of controlling the process of ammonia synthesis. Vestnik TGTU [Transactions of the TSTU], 2017, vol. 23, no. 4, pp. 572-580 (in Russ.).

[6] Omorov T.T., Kozhekova G.A., Zholdoshov T. Synthesis method of automated regulators for non-stationary linear multidimensional systems. Izvestiya Natsional'noy Akademii nauk Kyrgyzskoy Respubliki, 2012, no. 3, pp. 90-93 (in Russ.).

[7] Bayas M.M., Dubovoy V.M. Efficient resources allocation in technological processes using genetic algorithm. Middle East J. Sci. Res., 2013, no. 14 (1), pp. 1-4.

[8] Sun Q.-M., Yan H.-S. Multidimensional Taylor network optimal control of MIMO nonlinear systems without models for tracking by output feedback. Math. Probl. Eng., vol. 2017, art. 1548095. DOI: 10.1155/2017/1548095

[9] Muromtsev Yu.L. Full analysis methodology of energy-saving control on multidimensional objects. Sistemy upravleniya $i$ informatsionnye tekhnologii, 2008, no. 2-3, pp. 364-369 (in Russ.).

[10] Muromtsev D.Yu., Gribkov A.N., Kotok A.Yu., et al. [Methodology for developing destabilizing control systems of technological objects working under variable load]. Matematicheskie metody v tekhnike i tekhnologiyakh. Sb. tr. mezhdunar. nauch. konf. T. 2 [Mathematical methods in technique and technology. Proc. Int. Sci. Conf. Vol. 2]. St. Petersburg, Izd-vo Politekh. Univ. Publ., 2017, pp. 22-26 (in Russ.).

[11] Shamkin V.N., Muromtsev D.Yu., Gribkov A.N. Using destabilization control to improve the functioning of complex multidimensional technological objects on the time interval. JEAS, 2017, vol. 12, no. 24, pp. 7198-7217.

[12] Muromtsev D.Yu., Gribkov A.N., Tyurin I.V., et al. Formalised statement of a problem dealing with energy-efficient destabilisation control of multidimensional technological objects for the case of repeated adjustment of expected performance over a long period of time. Vestn. Mosk. Gos. Tekh. Univ. im. N.E. Baumana, Priborostr. [Herald of the Bauman Moscow State Tech. Univ., Instrum. Eng.], 2018, no. 5, pp. 99-115 (in Russ.). DOI: 10.18698/0236-3933-2018-5-99-115

Muromtsev D.Yu. - Dr. Sc. (Eng.), Professor, Vice-Rector for Scientific Innovation Activity, Professor, Department of Design of Radioelectronic and Microprocessor Systems, Tambov State Technical University (Sovetskaya ul. 106, Tambov, 392000 Russian Federation).

Gribkov A.N. - Dr. Sc. (Eng.), Assoc. Professor, Head of Department of Power Supply for Enterprises and Thermal Engineering, Tambov State Technical University (Sovetskaya ul. 106, Tambov, 392000 Russian Federation).

ISSN 0236-3933. Вестник МГТУ им. Н.Э. Баумана. Сер. Приборостроение. 2019. № 1 
D.Yu. Muromtsev, A.N. Gribkov, I.V. Tyurin, V.N. Shamkin

Tyurin I.V. - Cand. Sc. (Eng.), Assoc. Professor, Department of Design of Radioelectronic and Microprocessor Systems, Tambov State Technical University (Sovetskaya ul. 106, Tambov, 392000 Russian Federation).

Shamkin V.N. - Dr. Sc. (Eng.), Assoc. Professor, Professor, Department of Design of Radioelectronic and Microprocessor Systems, Tambov State Technical University (Sovetskaya ul. 106, Tambov, 392000 Russian Federation).

\section{Please cite this article as:}

Muromtsev D.Yu., Gribkov A.N., Tyurin I.V., et al. Theoretical Justification for the Solution of the Problem of Destabilization Energy-Saving Control of Multidimensional Technological Objects Operating in Variable Performance Conditions over a Long Time Interval. Herald of the Bauman Moscow State Technical University, Series Instrument Engineering, 2019, no. 1, pp. 109-128.

DOI: 10.18698/0236-3933-2019-1-109-128

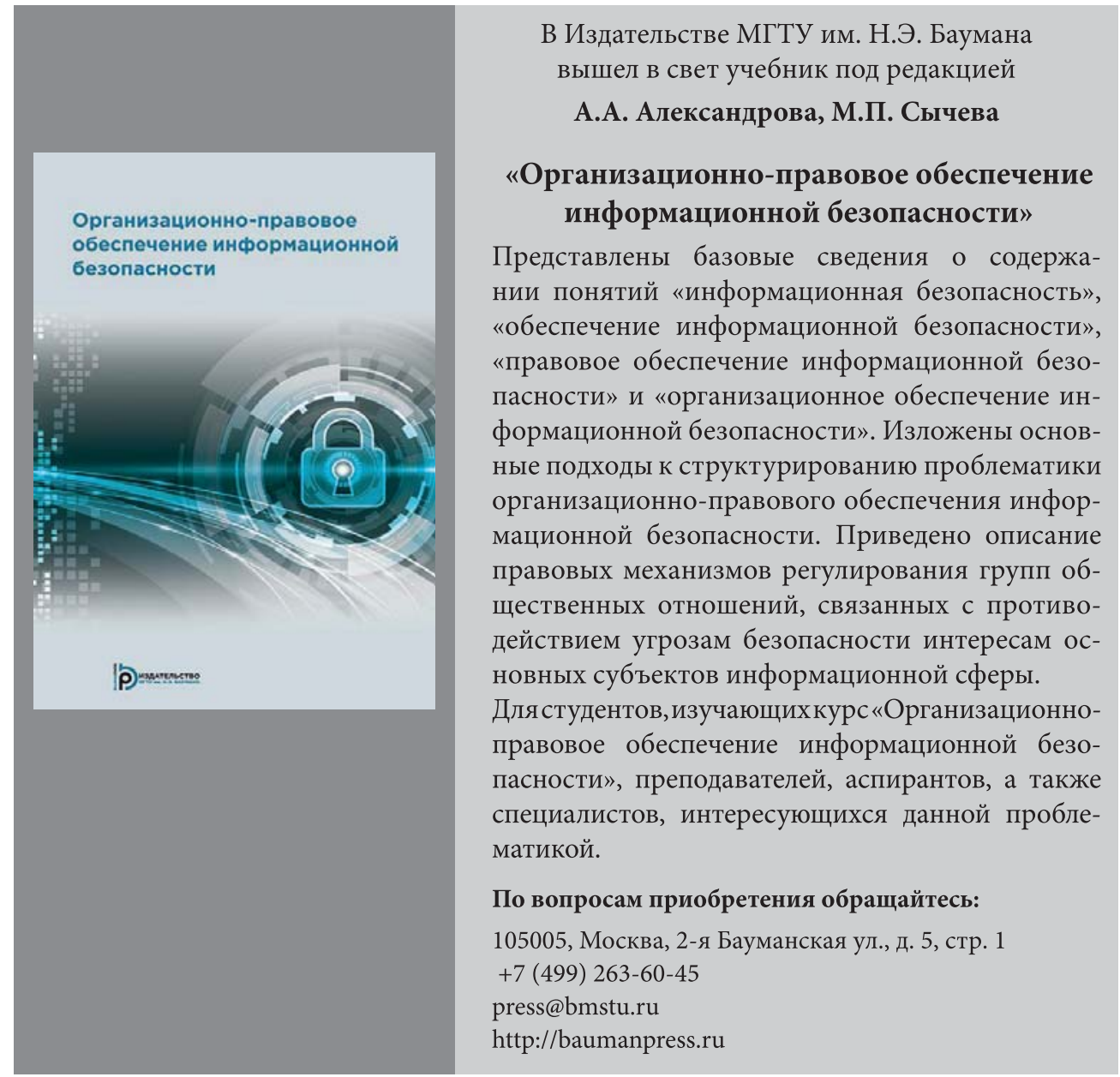

